Sādhanā Vol. 40, Part 3, May 2015, pp. 787-802. (C) Indian Academy of Sciences

\title{
A numerical study on dynamics of spray jets
}

\author{
AVICK SINHA*, SRIDHAR BALASUBRAMANIAN \\ and SHIVASUBRAMANIAN GOPALAKRISHNAN
}

Department of Mechanical Engineering, Indian Institute of Technology Bombay, Mumbai 400076, India

e-mail: avick.sinha@iitb.ac.in

MS received 18 May 2014; revised 21 January 2015; accepted 6 March 2015

\begin{abstract}
The study of flow characteristics of spray jets in an injector nozzle, solgel process is very critical for scientific studies. In this communication, we report results from a numerical modeling of spray jet dynamics and its breakup. The nature of instability depends on the density of the jet fluid and the ambient fluid and also on the velocity of the jet. The present work is motivated by the lack of quantitative measurement to explain the nature of instability of a vertically descending jet into a stagnant medium. In order to capture the sharp gradient between the interfaces, modified Volume of Fluid, using an extra compression term is used. The velocity profiles and spread angle are measured to quantitatively explain the mixing and growth of the instability in such complex multiphase flows.
\end{abstract}

Keywords. Jet; instability; breakup; volume of fluid.

\section{Introduction}

The study of a liquid jet exiting into another stagnant fluid is of interest in modeling the breakup of the jet and to understand the ensuing instability that governs the growth and mixing characteristics of flow encountered in many situations of engineering. Liquid jet coming out in a lighter medium, forms a freely falling stable jet, which accelerates and contacts with axial distance under the effect of gravity. Liquid jet injected into liquid has been studied for a long time both experimentally and numerically, and numerous literatures are available on the topic. Briefly, a few important findings of dynamics of spray jet breakup are documented here. Plateau (1873) proposed that in order to achieve a state of minimum surface energy, a round jet of diameter D must break down into equal segments of length 4.5D. Rayleigh (1879) found that breakup of a round jet in a different medium is caused by the hydrodynamic instability due to surface tension and occurs at relatively low jet Reynolds number. At large Reynolds numbers it is observed that the jet behaves in a wavy manner due to induction of aerodynamic effect on jet. Weber (1931) observed that liquid viscosity has stabilizing effect on jet which reduces the chances of breakup

*For correspondence 
and increases the size of the droplet. Taylor \& Hoyt (1977a) found that density of the stagnant fluid has an important effect on jet breakup. Chaudhary \& Maxworthy (1980a) conducted experiment on jet injected along the gravity and observed formation of satellite drops which get merged with the parent drop. Lin \& Reitz (1998) describes the various breakup regimes of a liquid jet when injected into a stationary gas. Based on the different combinations of liquid inertia, aerodynamic forces and surface tensions four different regimes have been identified. The regimes have been divided based on the difference in the appearance of the jet. The regimes are as follows: (a) the Rayleigh regime, where the jet diameter is smaller than the drop diameters, (b) the first wind induced regime, where the jet and drops diameter are of the same order, (c) the second wind induced regime, where the jet diameter is larger than the drop diameter and (d) atomization regime where drop sizes are negligible compared to jet diameter.

Leroux et al (1996) found another convenient method for categorizing different breakup regimes by considering the coherent portion length of the liquid jet or the unbroken length, as a function of exit velocity of jet. It is observed that beyond the region where the flow is dripping, the breakup length first increases linearly with the increase in velocity and then start decreasing after reaching a maximum. The diameters of the droplets dripping from the jet are comparable to the diameter of the jet. These correspond to the Rayleigh and first wind induced breakup regimes. Homma et al (2006) observed that when a Newtonian jet is injected at high velocity into another immiscible fluid, a transition from $2 \mathrm{D}$ axisymmetric to $3 \mathrm{D}$ flow takes place.

Although the breakup of a jet in a different medium has been studied for many years, there are still some niche areas related to understanding of jet disintegration for a downward vertically falling jet emanating into another medium. For example, the effects of turbulence at the inlet, the effect of density ratio between the jet and the stagnant medium, and the thermodynamic states of both gas and liquid have not been studied adequately. Moreover, the flow characteristics of a lighter fluid emanating into a heavier fluid have not been studied adequately. The present work seeks to gain a fundamental understanding of the instability of a vertically descending jet and the associated entrainment dynamics. The present study is carried out by the transient filling of a fluid into a chamber initially containing another fluid with a lower or a higher density. In such problems, sharp gradient exists between the fluid interfaces which is captured using a powerful but simple method that is based on the basis of fractional volume of fluid (VOF) (Nicholos \& Hirt 1981) with an extra compression term (Wardle \& Weller 2013). This computational fluid dynamics model is found to be more efficient and flexible compared to other methods for solving free boundary problems. In real flows, all scales of motion will affect the flow, hence to take into account the effect of small scales of motion; the RNG $k-\epsilon$ model (Jones 1972) is used. For flows where a lighter fluid enters into a heaver medium, buoyancy needs to be considered hence standard $k-\epsilon$ turbulence model (Yakhot 1992) is used because in RNG $k-\epsilon$ model buoyancy is neglected.

The format of this paper is as follows. In Section 2, the governing equations and numerical approach used for solving the present problem are given. Computational domain and Boundary conditions are given in Section 3. Results and discussion are discussed in Section 4, which is further subdivided into three parts. In the first part, validation of the code is shown. In second part, the study of a jet into a lighter medium is carried out, followed by the study of jet into a heavier medium is carried out in Section 4.2 and 4.3 respectively. Section 5 summarizes the results and points out the issues that remain to be investigated. 


\section{Governing equations and numerical approach}

For multiphase flow, the algorithm used to solve for two fluid equations is an algorithm developed by Wardle \& Weller (2013), which uses an interface compression technique to resolve some of the fundamental problems of the traditional VOF interface compression methods. Only one mass and momentum conservation equations, which is same for both the phases is solved. Based on the volume fraction of both fluids in one cell, weighted average is done to calculate the physical properties of a fluid. The non-dimensional continuity equation in tensor takes the form:

$$
\frac{\partial U_{i}^{*}}{\partial X_{i}^{*}}=0
$$

where the velocity scale is non-dimensionalized using velocity at the inlet of the nozzle $\left(U_{\mathrm{o}}\right)$ and the length scale is non-dimensionalized using diameter (d) of the nozzle inlet.

$$
U_{i}^{*}=\frac{U_{i}}{U_{o}} \text { and } X_{i}^{*}=\frac{X_{i}}{d}
$$

The non-dimensional momentum equation is tensor form given as

$$
\rho^{*} U_{j}^{*} \frac{\partial U_{i}^{*}}{\partial X_{j}^{*}}=\frac{\rho^{*}}{F r^{2}}+\frac{\partial}{\partial X_{j}^{*}}\left[-P^{*} \delta_{i j}+\mu\left\{\frac{\partial U_{i}^{*}}{\partial X_{j}^{*}}+\frac{\partial U_{j}^{*}}{\partial X_{i}^{*}}\right\}-\frac{1}{W e} k \tilde{n}\right.
$$

where the density has been non-dimensionalized using the heavier fluid density, Pressure has been non-dimensionalized using density and velocity, Fr is the Froude number and We is the Weber number.

$$
\rho^{*}=\frac{\rho}{\rho_{\text {heavy fluid }}}, P^{*}=\frac{P}{\rho U_{o}^{2}}, t^{*}=\frac{t U_{o}}{d}, F r=\frac{U_{o}}{\sqrt{g d}} \text { and } W e=\frac{\rho U_{o}^{2} d}{\sigma}
$$

where $\sigma$ is the surface tension force.

$$
n \text { is the unit vector, which is normal to the interface, } \quad n=\frac{\nabla \alpha}{|\nabla \alpha|}
$$

where $\alpha$ is the volume fraction

$$
k \text { is the curvature of the interface, } \quad k=\nabla . n
$$

The force due to surface tension $\left(\mathrm{F}_{\mathrm{S}}\right)$ is incorporated in the code, using the following equation:

$$
F_{s}=\sigma k n
$$

The fluid volume in a cell is calculated as,

$$
V_{\text {fluid }}=\alpha V_{c}
$$

where $\mathrm{V}_{\mathrm{C}}$ is the volume of the computational cell. Alpha $(\alpha)$ is a scalar indicator function that varies between 0 and 1 and is used to distinguish between two different fluids. In this method, 
the equations of flow are directly volume averaged in order to obtain single set of equations and the volume fraction is used to track the interface.

- $\alpha=1$ means control volume consists of only phase 1 .

- $\alpha=0$ means control volume consist of phase 2 .

- $0<\alpha<1$ indicates the present of interface.

The equations of flow are volume averaged using an averaged volume which is smaller than the volume of the drop in the simulation. The interface compression is obtained by an additional artificial compression term to the transport equation (Wardle \& Weller 2013), details about the extra compression term and $\overline{u_{c}}$ is given in the (Wardle \& Weller 2013)

$$
\frac{\partial \alpha}{\partial t}+\overline{u_{J}} \cdot \nabla \alpha+\nabla \cdot\left(\overline{u_{c}} \alpha(1-\alpha)\right)=0
$$

where $\overline{u_{c}}$ is the compression velocity, applied normally to the interface, suitable to compress the volume fraction field. This helps in maintaining a sharp interface. The term $\alpha(1-\alpha)$ will make sure that the extra artificial term in the volume fraction transport equation is engaged only at the interface of two fluids.

At any point in the numerical domain, density is calculated as the weighted average of the volume fraction of the fluids.

$$
\rho=\alpha \rho+(1-\alpha) \rho
$$

The transport equations for the dissipation rate $\epsilon$ and turbulence kinetic energy $k$ are solved and are shared throughout the domain by the two phases. The renormalized $k-\epsilon$ model is chosen for a flow, when the density of the jet coming out is more than the density of the fluid, in which small scales motion is of importance. The mathematical expression of this model is given below.

For turbulent kinetic energy, $k$ :

$$
\frac{\partial(\rho k)}{\partial t}+\frac{\partial\left(\rho k u_{i}\right)}{\partial x_{i}}=\frac{\partial}{\partial x_{j}}\left[\left(\mu+\frac{\mu_{t}}{\sigma_{k}}\right) \frac{\partial k}{\partial x_{j}}\right]+P_{k}-\rho \in
$$

For dissipation, $\epsilon$

$$
\frac{\partial(\rho \in)}{\partial t}+\frac{\partial\left(\rho \in u_{i}\right)}{\partial x_{i}}=\frac{\partial}{\partial x_{j}}\left[\left(\mu+\frac{\mu_{t}}{\sigma_{\epsilon}}\right) \frac{\partial k}{\partial x_{j}}\right]+C_{1 \in \frac{\epsilon}{k}} P_{k}-C_{2 \in \rho} \frac{\epsilon^{2}}{k}
$$

where $\sigma_{k}=0.7194, \sigma_{\epsilon}=0.7194, C_{1 \epsilon}=1.42, C_{2 \epsilon}=1.68$ are the standard values of the normalized $k-\epsilon$ model.

The standard $k-\epsilon$ model is used when the buoyancy effect dominates. For a flow, where a lighter jet penetrates into a heavier medium buoyancy effect will be important.

For turbulent kinetic energy, $k$ :

$$
\frac{\partial(\rho k)}{\partial t}+\frac{\partial\left(\rho k u_{i}\right)}{\partial x_{i}}=\frac{\partial}{\partial x_{j}}\left[\left(\mu+\frac{\mu_{t}}{\sigma_{k}}\right) \frac{\partial k}{\partial x_{j}}\right]+P_{k}-\rho \in-Y_{M}+S_{k}+P_{b}
$$

For dissipation, $\epsilon$

$$
\frac{\partial(\rho \in)}{\partial t}+\frac{\partial\left(\rho \in u_{i}\right)}{\partial x_{i}}=\frac{\partial}{\partial x_{j}}\left[\left(\mu+\frac{\mu_{t}}{\sigma_{\epsilon}}\right) \frac{\partial k}{\partial x_{j}}\right]+C_{1 \in \frac{\epsilon}{k}}\left(P_{k}-C_{3 \in} P_{b}\right)-C_{2 \in \rho} \frac{\epsilon^{2}}{k}+S_{\in}
$$


where $C_{1 \epsilon}=1.44, C_{2 \epsilon}=1.92, C_{3 \epsilon}=-0.33$ are the standard values for $k-\epsilon$ model. For the details description of each individual term, refer Jones (1972) and Yakhot (1992).

The equations are solved on finite volume grid using iterative scheme, PISO (Yin \& Chow 2003) to solve the $\mathrm{N}-\mathrm{S}$ equation. The continuity equation and advection terms are solved implicitly, the body force term is solved explicitly. The solver calculates the CFL number and based on this, it calculates a new time step. The stopping criterion is based on the continuity error having convergence criteria of $10^{-6}$ before it writes out the data. The numerical model is implemented using a framework called OpenFOAM.

\section{Computational domain and boundary conditions}

The geometry of the numerical domain along with the boundary conditions is shown in figure 1 . For simplicity a 2-D numerical domain is considered for the study. The fluid considered obeys the laws of Newtonian mechanics and is assumed to be incompressible and isothermal. The jet from the nozzle comes out into a chamber which is at atmospheric condition. The inlet velocity to the nozzle is kept fixed, the walls are maintained at no slip conditions and the top of the chamber is open to the atmosphere, so that the excess fluid inside the container can move out and a constant mass is maintained inside the chamber.

Initially $(\mathrm{t}=0)$, the velocities at the inlet of the nozzle are (a) for lighter into heavier liquid $0.05 \mathrm{~m} / \mathrm{s}$ to $0.2 \mathrm{~m} / \mathrm{s}$ in step of $0.05 \mathrm{~m} / \mathrm{s}$ and (b) for heavier to lighter fluid flow $-0.2 \mathrm{~m} / \mathrm{s}$ to 0.8 $\mathrm{m} / \mathrm{s}$ in step of $0.2 \mathrm{~m} / \mathrm{s}$ respectively. The outlet pressure is fixed at 1 bar for all cases. The velocity of the wall of the nozzle and the container is always zero $\mathrm{m} / \mathrm{s}$.

For this problem, fine uniform mesh of 1, 24,000 nodes are used after checking grid convergence.

\section{Results and discussion}

\subsection{Validation}

To validate the numerical results, jet shape function (JSF) (Senechenko \& Bohr 2005), which represents the relationship between the axial distance from the nozzle outlet and the jet radius

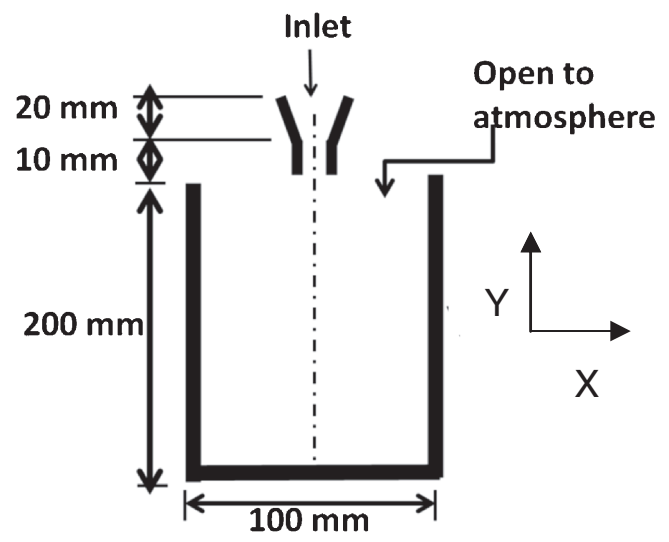

Figure 1. Schematic diagram of the computational domain. 
(as shown in figure 2) has been compared with the modified analytical form of JSF proposed by Adachi (1987). The mathematical form of the JSF derived by Adachi (1987) is given as

$$
\bar{z}=F r\left(\frac{1}{\overline{r^{4}}}-m\right)-\frac{n}{B o}\left(\frac{1}{\bar{r}}-1\right)
$$

where

$$
\bar{z}=\frac{z}{R} \text { and } \bar{r}=\frac{r}{R}
$$

$z$ is the axial distance from the exit of the nozzle, $R$ is the radius of the nozzle, $r$ is the jet radius. Fr is Froude number and Bo is the Bond number. The value of $m$ and $n$, which are model parameters, is given as 1 and 12 respectively as given in (Massalha \& Digilov 2013).

$$
B o=\frac{4 \rho g R^{2}}{\sigma}
$$

where $V_{0}$ is the exit velocity of the nozzle at the nozzle exit and $\sigma$ is the surface tension.

The first term in Eq. 15 represents the effects of gravity while the second term represents the effect of surface tension. For validation, isothermal liquid jet of density $1000 \mathrm{~kg} / \mathrm{m}^{3}$ issuing out from a nozzle of $10 \mathrm{~mm}$ diameter with exit average velocity of $0.39 \mathrm{~m} / \mathrm{s}$ into air has been

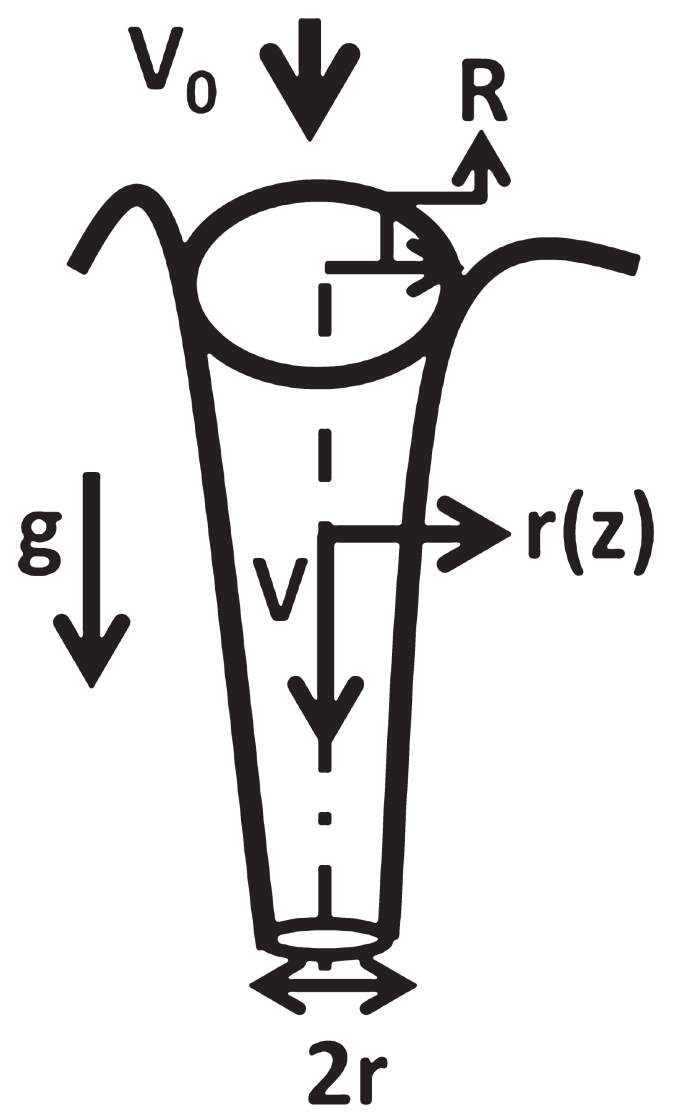

Figure 2. Schematic diagram of fluid jet showing relevant variable. 


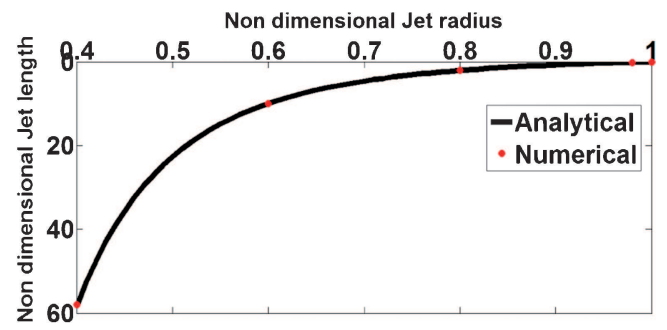

Figure 3. Comparison of numerical with analytical results given by Eq. 15 .

considered. It is observed that the results obtained numerically agree well with the analytical solution given by Eq. 15 as shown in figure 3 .

The results are further divided into two sections. In the first section, the flow characteristic of a heavier density jet into a lighter medium is studied and a flow characteristic of a lighter jet into a heavy density medium is studied in the second section.

For both the cases, the time scale and the length scale in the axial direction are nondimensionalized and are given as

$$
t^{*}=U_{0} t / d \text { and } y^{*}=y / d
$$

where $U_{\mathrm{o}}$ is the initial inlet velocity to the nozzle, $t$ is the time after which the flow has started, $d$ is the diameter of the nozzle inlet, $t^{*}$ and $y^{*}$ are the non-dimensional time and length scale respectively. $y^{*}$ zero indicates inlet to the nozzle, while $y^{*}=11.5$ denotes the bottom wall of the container.

\subsection{Flow of a heavier density jet into a lighter medium}

For this case, water is considered as the fluid entering the tank which is initially filled with air. This gives a density ratio of 1000 . The inlet velocities to the nozzle are $0.05,0.1,0.15$ and $0.2 \mathrm{~m} / \mathrm{s}$. The minimum Reynolds number of the jet based on the minimum inlet velocity is 1000 , which is turbulent in nature. The Reynolds numbers based on above velocities are 1000, 2000, 3000 and 4000 respectively.

4.2a Volume fraction: The temporal variation of the injected phase for $R e=1000$ is shown in figure 4. It is observed that at $t^{*}=0.45$ from the start of filling the process the jet of water stream breaks up as shown in figure 4 a.

It is observed that beads of water droplets starts forming once the initial water droplet breaks down due to higher shear stress compared to surface tension forces as shown in figure $4 \mathrm{~b}$.

Under the influence of gravity, the jet narrows down with the increase in distance from the nozzle. The diameter of the primary droplet increases as it gets detached from the jet. Here, primary droplet refers to the first droplet that gets detached from the jet due to dominant shear stress. The increase in droplet diameter is due to surface tension of the fluid. The shape of the droplet changes from cup-shaped to elliptic and finally to circle, which is the most stable state for the droplet to be in equilibrium. Four modes of drop formation are used to characterize the unsteady interface dynamics - only Primary droplet (P), Primary with satellite droplets (PS), Random (R) and no breakup of the jet (NJ). As observed in figure 4, the mode of droplet formation changes from $P$ (figure $4 a$ ) to $R$ (figure $4 d$ ) with the increase in time. The small droplet 


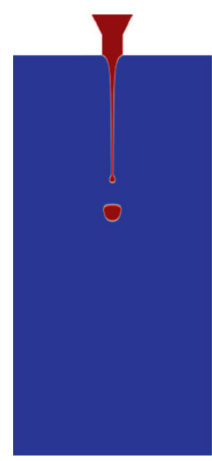

(a)

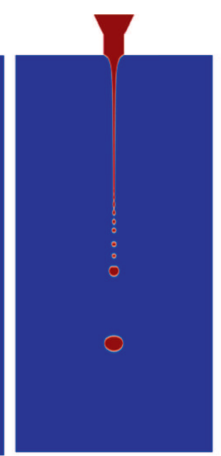

(b)

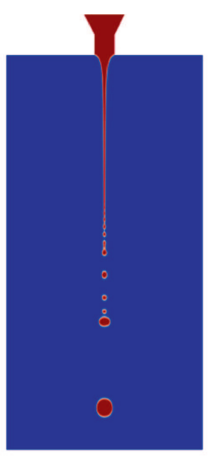

(c)

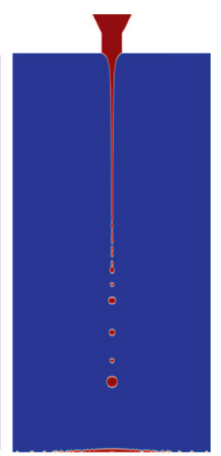

(d)

Figure 4. Field alpha for $R e=1000$ after $t^{*}=$ (a) 0.45 (b) 0.575 (c) 0.625 and (d) 0.675 from start respectively.

which is formed after the primary droplets and is between the primary droplet and the jet tip is referred to as satellite drops. The main reason for PS mode is due to the recoiling of the jet. Random here refers to as formation of droplet of any shape.

Second wind induced regime instability is observed when the primary drop with diameter smaller than the nozzle outlet diameter $(10 \mathrm{~mm})$ gets detached from the jet with a low velocity inlet to the nozzle (figure 4b). With the passage of time, due to surface tension effect, the droplet diameter becomes almost close to the nozzle diameter. This regime refers to as the first wind induced regime.

For $R e=2000$, it is observed that with the increase in the inlet velocity to the nozzle, the size of the primary droplet increases due to the higher axial momentum, this squeezes more fluid into the droplet (figure 5). Moreover due to higher momentum, the jet covers more distance before the shear thickening effect can dominates. The jet stretches and narrows as it moved because of the effect of gravity. Based on the droplet diameter which is almost the same as the nozzle outlet diameter, first order induced regime is observed. Also, with the increase in velocity the time scale over which the formation of primary droplet takes place increases.

For $R e=3000$ and $R e=4000$, the above observations are not observed as shown in figure 6 . This is due to the high momentum of the jet which overcomes the aerodynamic and surface tension breakup. The jet disintegrates once it impinges on the bottom wall of the container. For higher momentum jet, we observed a NB mode of the jet.

4.2b Velocity profiles: The velocity profiles for different Reynolds number at different axial location is shown in figure 7. It is observed that with the increase in the axial distance, the jet diverges. This is due to the entrainment of surrounding fluid. The little humps for $R e=1000$ at an axial location of $y^{*}=6$ as shown in figure 7 a are due to the initiation of breakup of the jet at this particular location. The increase in the velocity of the jet with the increase in the downstream distance from the nozzle is due to the effect of gravity. A top hat profile is observed close to the nozzle exit for all Reynolds numbers, which confirms that the jet is turbulent at the nozzle exit. With the increase in the downstream distance, the jet maintains its top hat profile which shows that the jet is still turbulent.

The normalized velocity profile for all $R e$ at a particular downstream location is shown in figure 8 . The velocities for all Reynolds numbers are normalized by the inlet velocity to the 


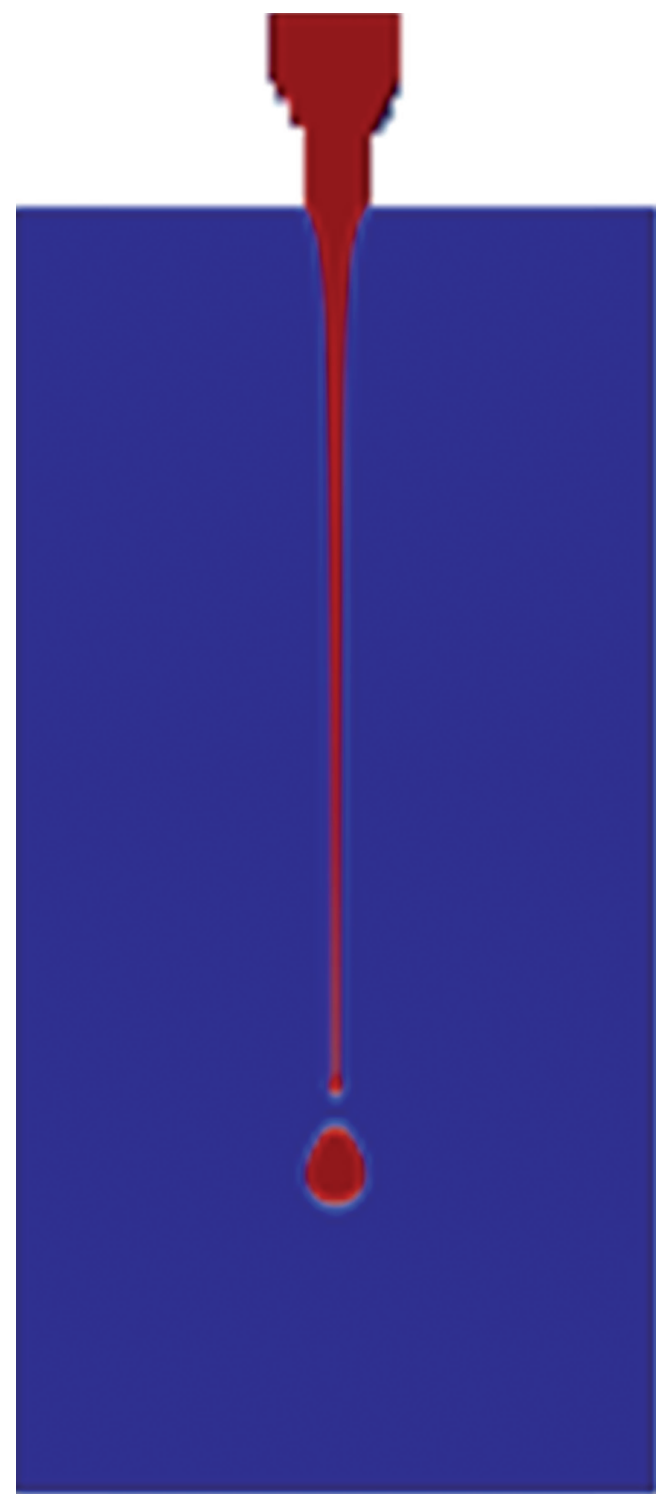

Figure 5. Field alpha for $R e=2000$ after $t^{*}=1$.

nozzle and the radial distance is normalized using the diameter of the nozzle inlet. It is observed that with the increase in the Reynolds number the jet spread is increasing. This is due to higher turbulent diffusivity which increases with the increase in velocity of the jet. The entrainment of the jet also increases with the increase in $R e$ due to higher turbulent diffusion.

4.2c Centreline velocity: The normalized centreline velocity profiles for different $R e$ are shown in figure 9. It is observed that with the increase in the downstream distance, the centreline velocity increases in a quadratic manner. This is consistent with the increase in velocity for 


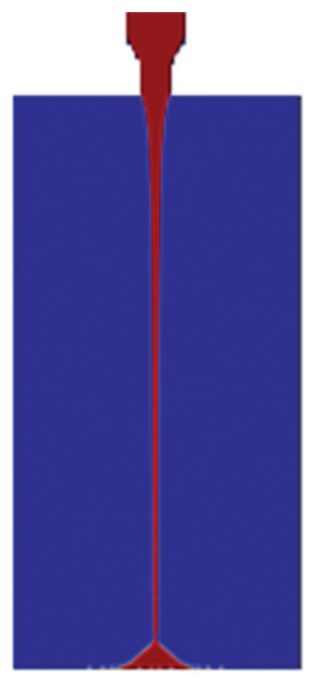

(a)

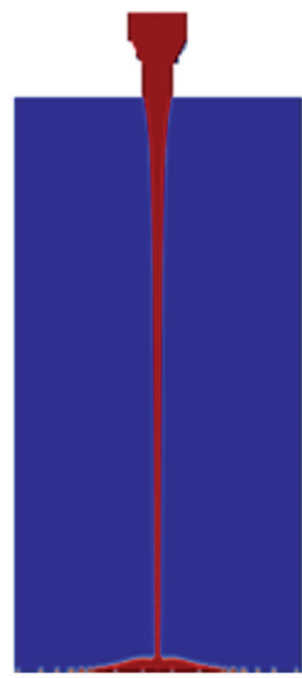

(b)

Figure 6. Field alpha for $R e=$ (a) 3000 and (b) 4000 .

a free falling jet, whose velocity is directly proportional to $\sqrt{2 g h}$. In contrast to horizontal jet, where the centreline velocity decreases linearly.

The centreline velocity for each $R e$ is normalized using the nozzle inlet velocity. The fluctuation in the centreline velocity for $R e=1000$ and $R e=2000$ near $y / d=8$ and 4 denotes the

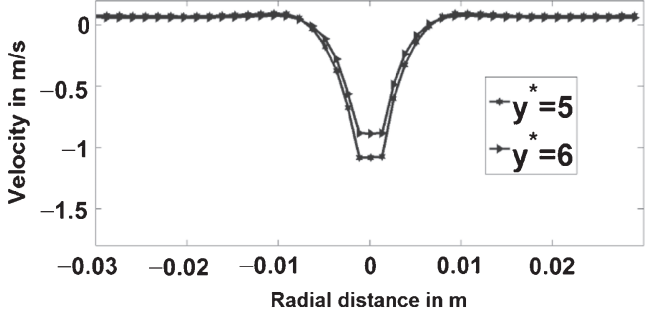

(a)

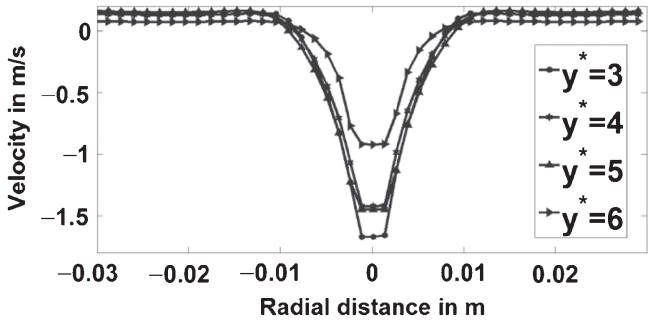

(c)

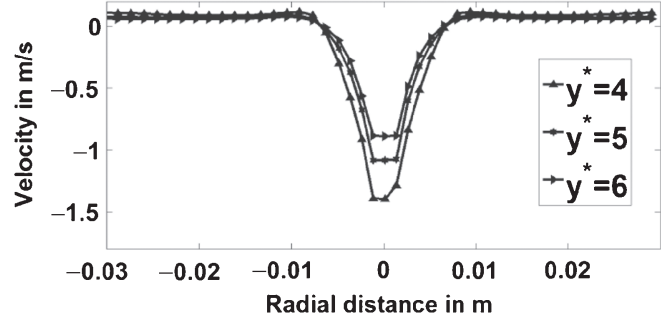

(b)

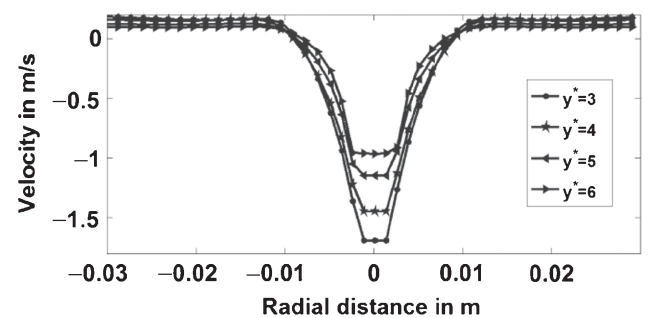

(d)

Figure 7. Velocity profiles at different axial locations for $R e=$ (a) 1000 (b) 2000 (c) 3000 and (d) 4000 . 


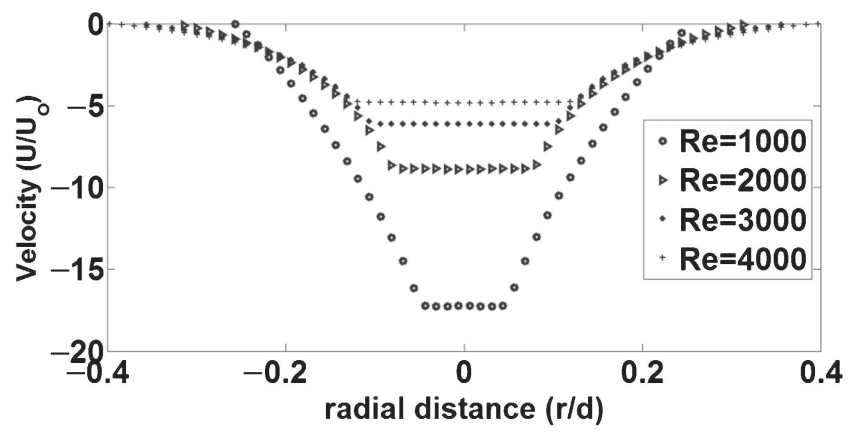

Figure 8. Normalized velocity profile for all $R e$ at $y^{*}=0.8$.

breakup of the jet at this location respectively. As a result, there is step jump towards zero velocity. Near $y / d=0$, which represents the bottom wall, the velocity decreases gradually because of the accumulation of the fluid in the bottom wall, as it impinges on the wall.

4.2d Spread angle: In figure 10, it can be seen that the jet cone angle increases with the increase in velocity. This is consistent with the velocity profiles in which the entrainment is more for higher velocity. The increase in the jet angle with velocity is quadratic in nature.

The cone angle is measured based on the jet semi-width, which the radial distance along which the velocity is one-fourth of the centreline velocity and axial location from the nozzle exit along which the jet semi-width radius is measured gives the semi-cone of the jet.

4.2e Effect of nozzle diameter: With the increase in nozzle diameter, the point of breakup of the jet moves further downstream as shown in figure 11. This is due to the increase in mass flow rate of jet which in turn increases the momentum of the jet.

\subsection{Flow of a lighter density jet into a heavier medium}

In this case, ethyl acetate having a density of $897 \mathrm{~kg} / \mathrm{m}^{3}$ comes out from the nozzle and into a tank, initially filled with water. Therefore the density ratio is 0.897 . The inlet velocities to the

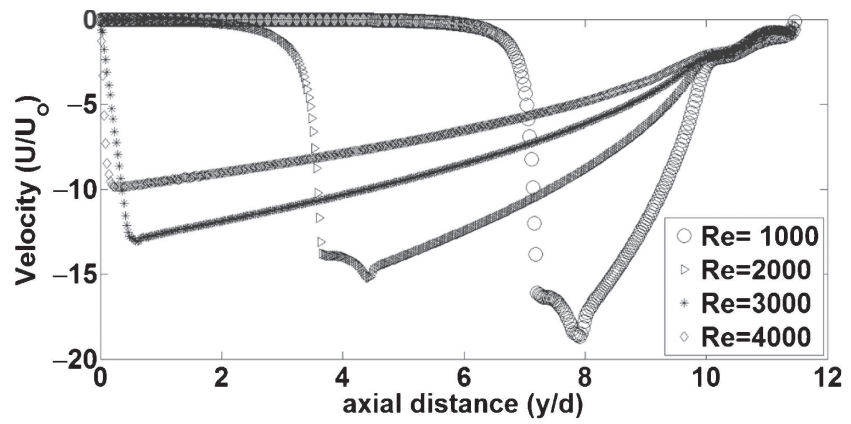

Figure 9. Normalized centreline velocity profiles for different $R e$. 


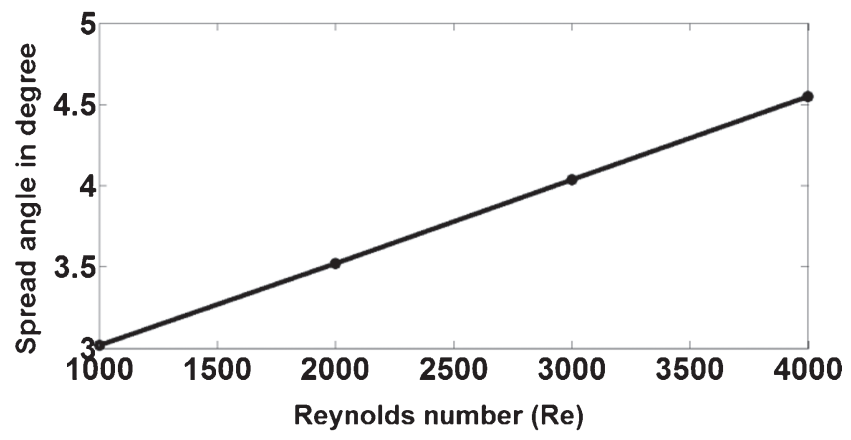

Figure 10. Jet spread angle.

nozzle considered are $0.2,0.4,0.6$ and $0.8 \mathrm{~m} / \mathrm{s}$. The minimum Reynolds number of the jet based on the minimum inlet velocity is 8,400 . If the inlet velocity to the nozzle is less than this because of the less momentum, it will not be able to displace the water. The Reynolds number based on above velocities is 8,400,16,800, 25,200 and 33,600. In this case, the red colour represents ethyl acetate, which correspond to $\alpha=1$ and $\alpha=0$ represent water.

4.3a Volume fraction: The volume fraction and vector diagram for different Reynolds number after $1 \mathrm{~s}$ from the start of filling process is shown in figure 12. It is observed that for $R e=4200$, the momentum is so less that the jet is unable to penetrate the heavier fluid rather the incoming fluid is oozes out from the top. This is consistent with the vector diagram (figure 13), which shows vector pointing outward (opposite the flow direction).

With the increase in the Reynolds number, the jet penetration increases but the amount of water trapped inside ethyl acetate also increases. This is due to higher shear stress and sharp gradients due to shear thinning at the interface of the two fluids. In vector diagrams, red colour represents highest velocity, while the blue colour represents lesser velocity compare to the velocity at the inlet of the nozzle.

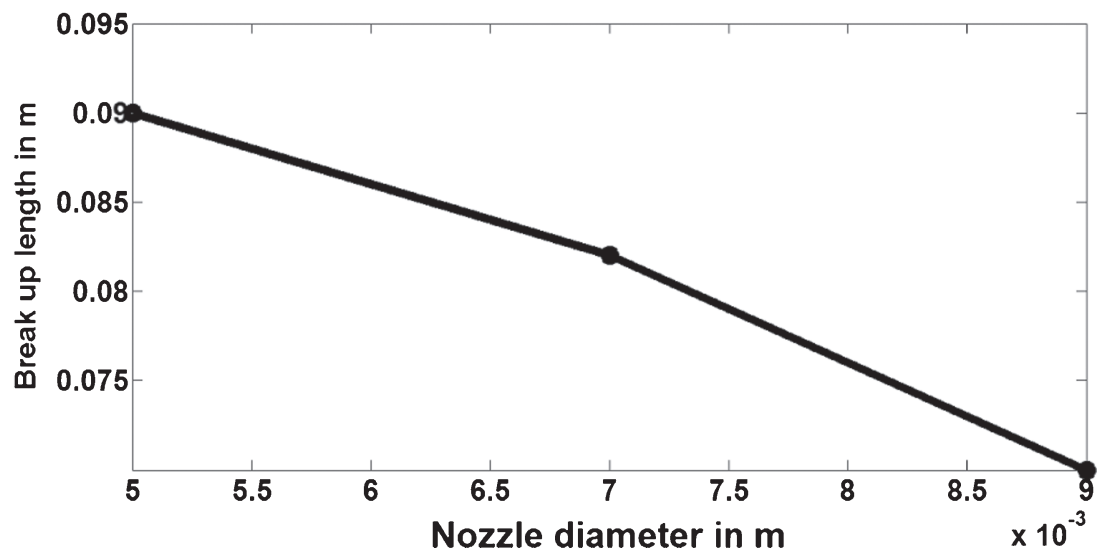

Figure 11. Effect of nozzle diameter. 


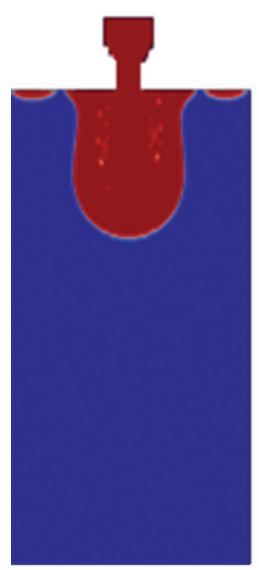

(a)

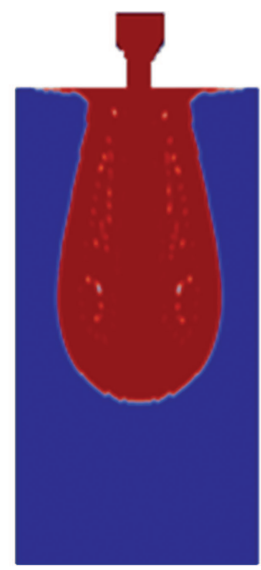

(b)

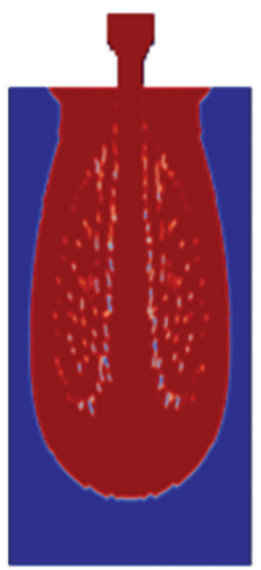

(c)

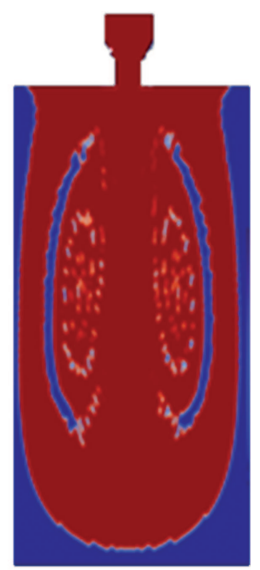

(d)

Figure 12. Field alpha after $1 \mathrm{~s}$ from the start for $R e=$ (a) 8,400 (b) 16,800 (c) 25,200 and (d) 33,600 .

4.3b Velocity profiles: The velocity profiles for different Reynolds number are shown in figure 14. It is observed that with the increase in the downstream distance from the nozzle the entrainment also increases. The decrease in velocity with the increase in the downstream distance is due to the reduction in momentum of the jet as it penetrates through the heavier fluid. For $R e=8,400$, at a downstream location of $y^{*}=5$, the velocities are quiet small and the velocity profile is fluctuating as shown in figure 14a. This is because the jet is unable to reach this particular location after $1 \mathrm{~s}$ from the start. The velocities at this particular location are due to velocity induced by the jet close to it. In the near field, a top hat profile is observed but with the increase in the downstream distance, the profile becomes Gaussian. This shows that the jet is developing and because of high viscosity of water the turbulence in the jet is decreasing. For higher Reynolds number jet, the double hump structure in near field zone develops due to sudden interaction between two fluids which gradually develops into a single peak curve at the far

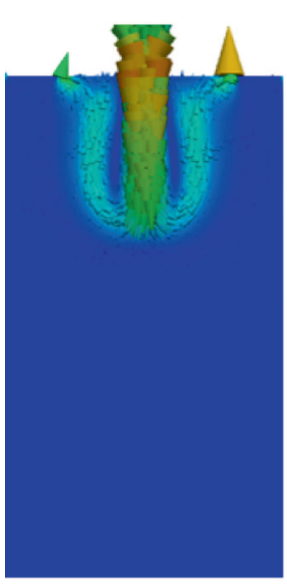

(a)

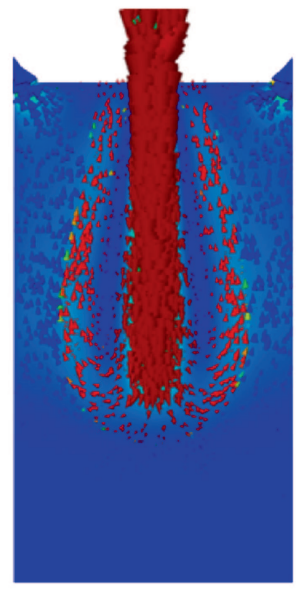

(b)

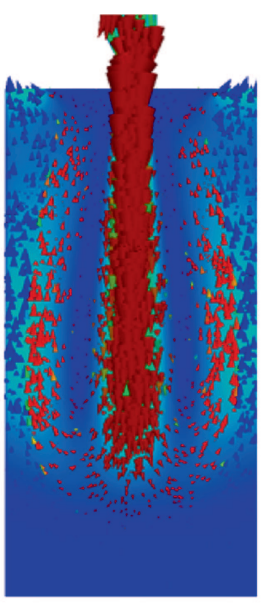

(c)

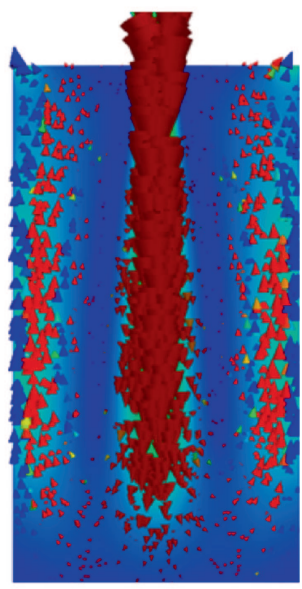

(d)

Figure 13. Vector diagram after $1 \mathrm{~s}$ from the start for $R e=$ (a) 8,400 (b) 16,800 (c) 25,200 and (d) 33,600 . 


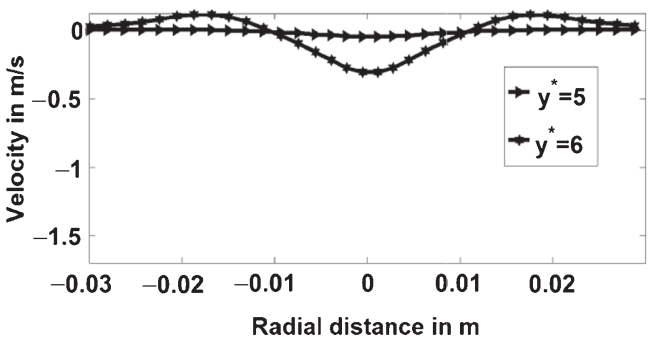

(a)

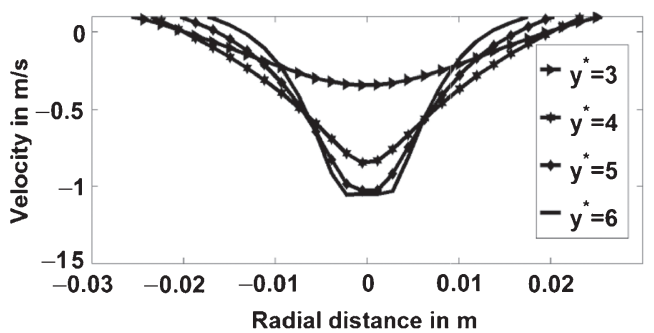

(c)

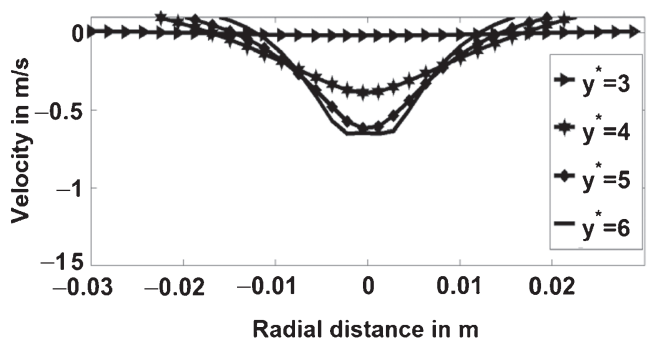

(b)

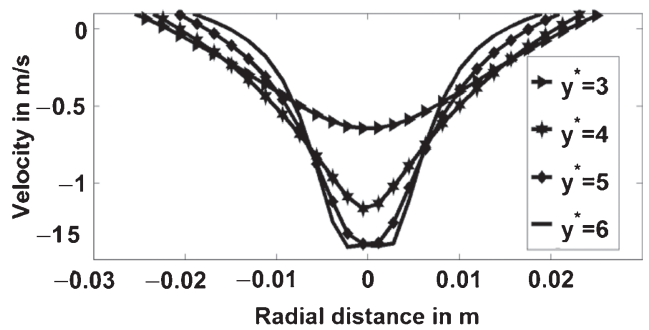

(d)

Figure 14. Velocity profiles at different axial locations for $\operatorname{Re}=$ (a) 4,200 (b) 8,400 (c) 12,600 and (d) 16,800 .

field. Absence of double hump structure for $R e=16,800$ is due to very less momentum of the jet. Therefore the moment the jet tries to penetrate the heavier fluid; there is very little shear layer formation between the two fluids.

The normalized velocity profile for all $R e$ at a downstream location of $y^{*}=6$ is shown in figure 15. The velocities for all Reynolds numbers are normalized by the nozzle inlet velocity and the radial distance is normalized using the outer diameter of the nozzle. It is observed that the except for $R e=8,400$, all the other curved coincides over each other near the centre, showing they have the similar characteristics. This may because the jet is yet to develop. In the far field, it is observed that with the increase in the velocity the jet spread also increases. This is due to turbulent diffusivity. For $R e=8400$, the momentum is so less and the viscosity is so high that it dampens the turbulent fluctuation and Gaussian profile is obtained. For other Reynolds number

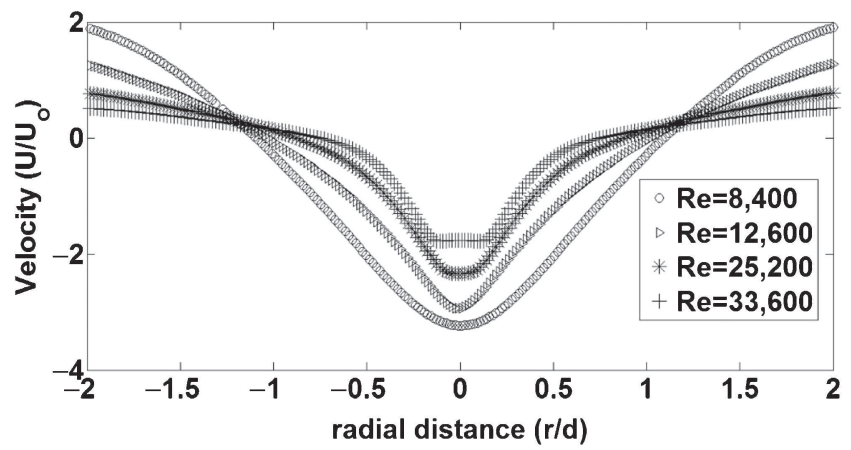

Figure 15. Normalized velocity profile at $y^{*}=0.8$. 


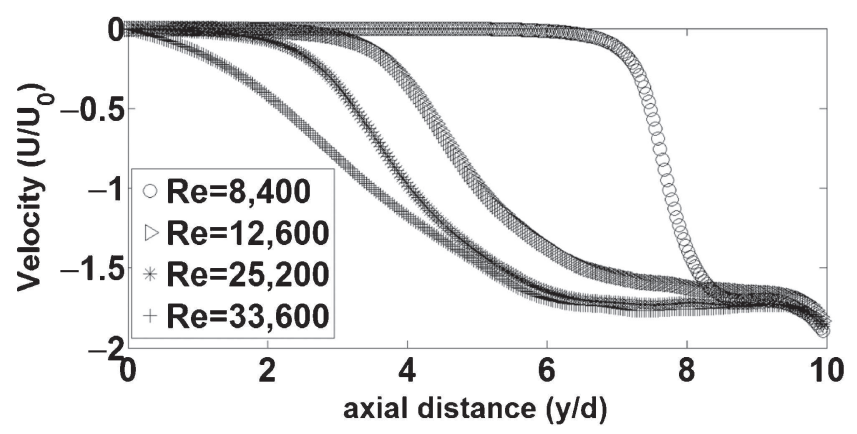

Figure 16. Normalized centreline velocity profiles for different $R e$.

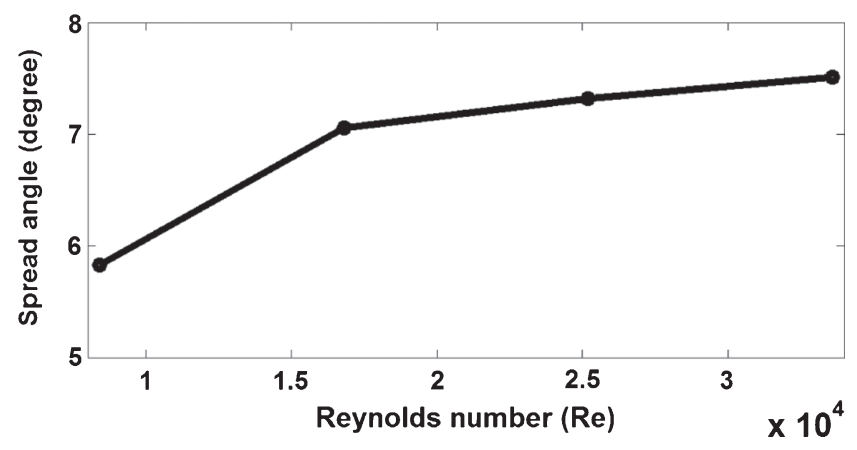

Figure 17. Jet spread angle.

at this location, top hat profile is obtained, showing that the jet is turbulent. This shows that high viscosity can act and stabilise the jet.

4.3c Centreline velocity: The normalized centreline velocity profiles for different $R e$ are shown in figure 16. It is observed that with the increase in the downstream distance, the centreline velocity decreases in a quadratic manner. This is because as the jet penetrates into a heavier medium, its momentum decreases as consequence the velocity also decreases.

The centreline velocity for each $R e$ is normalized using the nozzle inlet velocity and the axial distance is normalized using the outer diameter of the nozzle. It is observed from figure 16 that centreline velocity for $R e=8,400, R e=16,800, R e=25,200$ and $R e=33,600$ reaches to zero at $y / d=5,3,2.5$ and 1.25 because beyond this region there is no jet.

4.3d Cone angle: The jet cone angle increases with the increase in velocity. This may due to turbulent diffusion as shown in figure 17.

\section{Conclusions}

- The velocities and cone angle of the jet increase with increase in velocity and with the increase in downstream distance for density ratio more than 1. 
- In case of flows, where the density ratio is $<1$, it is observed that the velocity decreases with the increase in downstream distance also the velocity profile changes from top hat to Gaussian.

- For $R e \geq 3000$ no droplet formation takes place.

- When the density ratio is more/less than 1 , the centreline velocity increases/decreases in a quadratic manner.

- The penetration length varies linearly and quadratic manner when the density ratio is greater than and less than 1 respectively.

\section{References}

Adachi K 1987 Laminar jets of a plane liquid sheet falling vertically in the atmosphere. J. Non-Newtonian Fluid Mech. 24: 11-30

Chaudhary K C and Maxworthy T 1980a The nonlinear capillary instability of a liquid jet. Part 2. Experiments on jet behavior before drop formation. J. Fluid Mech. 96: 275-286

Homma S, Koga J, Matsumoto S, Song M and Tryggvason G 2006 Breakup mode of an axi-symmetric liquid jet injected into another immiscible liquid. Chem. Eng. Sci. 61: 3986-3996

Jones W P 1972 The prediction of Laminarization with a Two-Equation model of turbulence. J. Heat Mass Transfer 26: 566-575

Leroux S, Dumouche C and Ledoux M 1996 The stability curve of Newtonian liquid jet. At. Sprays 6: 62347

Lin S P and Reitz R D 1998 Drop and spray formation from a liquid jet. Annu. Rev. Fluid Mech. 30: 85-105

Massalha T and Digilov R M 2013 The shape function of a free falling laminar jet - Making use of Bernoulli's equation. Am. J. Phys. 81: 733-737

Nicholos B D and Hirt C W 1981 Volume of Fluid (VOF) Method for the dynamics of free boundaries. $J$. Computational Phys. 39: 201-205

Plateau D 1873 Stattique experimentale et theoretique des liquids Soumie Aux Seules forces moleicularies, Paris: Chanthier Vallars

Rayleigh L 1879 On the stability of jets. London Math. Soc. 10: 361-71

Senechenko S and Bohr T 2005 Shape and stability of aviscous thread. Phys. Rev. E 71: 056301-1-7

Taylor J J and Hoyt J W 1977a Waves on water jet. J. Fluid Mech.83

Wardle K E and Weller G H 2013 Hybrid multiphase CFD solver for coupled dispersed/segregated flows in liquid-liquid extraction. Int. J. Chem. Eng. 13

Weber C 1931 On the disruption of liquid jets. Z. Angrew, Math. Mech. 2(2)

Yakhot 1992 Development of turbulence models for shear flow by a double expansion technique

Yin R and Chow W K 2003 Comparison of four algorithms for solving pressure-velocity linked equations in simulating atrium fire. Int. J. Arch. Sci. 4(1): 24-35 